\title{
QUELQUES ASPECTS DU CYCLE BIOLOGIQUE DE PLECTROCNEMIA CONSPERSA (CURTIS, 1834) [Trichoptera, Polycentropodidae]
}

par H. TACHET.

Le cycle biologique des Trichoptères n'a été complètement décrit que pour un petit nombre d'espèces à larves de type éruciforme. C'est le cas notamment de quelques espèces de Limnephilidae [Novak 1959, Novak et Sehnal 1963], de Micropterna testacea (Limnephilidae) [Bournaud, travaux inédits], d'Enoicyla pusilla (Limnephilidae) [Kelner-Pillault 1960] et récemment de Drusus annulatus (Limnephilidae) [Gower 1966]. Le cycle vital de ces différentes espèces, à l'exception toutefois de Drusus annulatus, est relativement simple. Il n'y a qu'une seule génération par an. La majorité des individus sont, à un moment donné, au même stade.

Les larves campodéiformes et, en particulier, celles de la famille des Polycentropodidae ${ }^{1}$ présentent un cycle vital beaucoup plus complexe, comme le montrent en particulier les observations de Frost [1942] et Hynes [1961] pour Polycentropus flavomaculatus, Nielsen [1942] et Mackereth [1960] pour Plectrocnemia conspersa. En effet, en toutes saisons, on observe des individus à différents stades de développement, ce qui laisserait supposer l'existence d'au moins deux générations par an. Cette hypothèse ne peut que difficilement être vérifiée dans la nature en raison de la présence, pendant toute l'année, de larves au dernier stade larvaire et d'une longue période d'apparition des adultes; seule l'observation d'individus depuis l'éclosion jusqu'à la mue imaginale permettrait d'apporter quelques précisions sur ce point. C'est ce que nous nous sommes efforcés de faire avec Plectrocnemia conspersa.

\section{1. - MATÉRIEL ET METHODES.}

Les larves utilisées ont été récoltées dans le Mornantet à Mornant (Rhône). Elles sont isolées dans des bacs en matière plastique

1. D’après le «Trichopterorum catalogus », vol. III, 1962 : PolycentropodidaePsychomyidae de F. C. J. Fischer. 
transparente, ces derniers plongés dans un bain-marie dont la température varie, pendant l'année, de $9^{\circ} \mathrm{C}$ à $17^{\circ} \mathrm{C}$. Les adultes issus de ces larves sont placés dans une boîte en matière plastique à couvercle grillagé et dont le fond est recouvert de sable. Une coupelle pleine de sable maintenu humide permet de recueillir les pontes. Les larvules issues de ces pontes sont isolés à leur tour et observées quotidiennement.

Nous avons ainsi pu analyser les quatre phases du cycle de $P$. conspersa : vie larvaire, vie nymphale, vie imaginale et vie embryonnaire, et leur succession dans le temps. Nous avons effectué nos observations sur deux groupes de larves : groupe A et groupe B.

\section{2. - EVOLUTION DES LARVES DU GROUPE A.}

Ce groupe comprend 75 larves nées le $1^{\text {er }}$ novembre 1965; sur ce nombre, par suite de mortalité au niveau des premiers stades, 64 seulement arrivèrent au stade imaginal. Ces larves reçurent une fois par semaine une alimentation comprenant pour les stade I, environ $5 \mathrm{~cm}^{3}$ et pour les stades II et III, environ $10 \mathrm{~cm}^{3}$ d'un milieu de culture contenant des Copépodes Cyclopides, des Cladocères Chydorides et des Ostracodes. Les stades IV et $\mathrm{V}$ reçurent de 3 à 6 Tubificides.

\section{1. - La vie larvaire.}

C'est la partie la plus longue du cycle puisqu'elle dure en moyenne 280 jours. Complétant les données de Siltala [1907] et Nielsen [1942], qui décrivent le premier et le dernier stade lar-. vaire, nous avons pu constater que $P$. conspersa présente 5 stades larvaires. Nous avons rassemblé dans le Tableau I certaines caractéristiques de ces stades.

Tableau I. - Largeur de la capsule céphalique

et durée moyenne des stades larvaires des larves de $P$. conspersa.

\begin{tabular}{|c|c|c|c|c|}
\hline \multirow{2}{*}{ Stade } & \multicolumn{2}{|c|}{$\begin{array}{l}\text { Largcur de la capsule } \\
\text { céphalique }\end{array}$} & \multicolumn{2}{|c|}{$\begin{array}{l}\text { Durée moyenne } \\
\text { du développement larvaire } \\
\text { pour chaque stade }\end{array}$} \\
\hline & $\begin{array}{l}\text { Valeur } \\
\text { moyenne }\end{array}$ & Ecart type & $\begin{array}{l}\text { Valeur } \\
\text { moyenne }\end{array}$ & Ecart type \\
\hline I & $0,25 \mathrm{~mm}$ & $\sigma= \pm 0,01$ & 25 jours & $\sigma= \pm 6$ \\
\hline I1 & $0,41 \mathrm{~mm}$ & $\sigma= \pm 0,03$ & 37 jours & $\sigma= \pm 13$ \\
\hline III & $0,78 \mathrm{~mm}$ & $\sigma= \pm 0,05$ & 89 jours & $\sigma= \pm 16$ \\
\hline IV & $1,44 \mathrm{~mm}$ & $\sigma= \pm 0,07$ & 40 jours & $\sigma= \pm 7$ \\
\hline $\mathrm{V}$ & $2,35 \mathrm{~mm}$ & $\boldsymbol{\sigma}= \pm 0,15$ & 90 jours & $\sigma= \pm 16$ \\
\hline
\end{tabular}




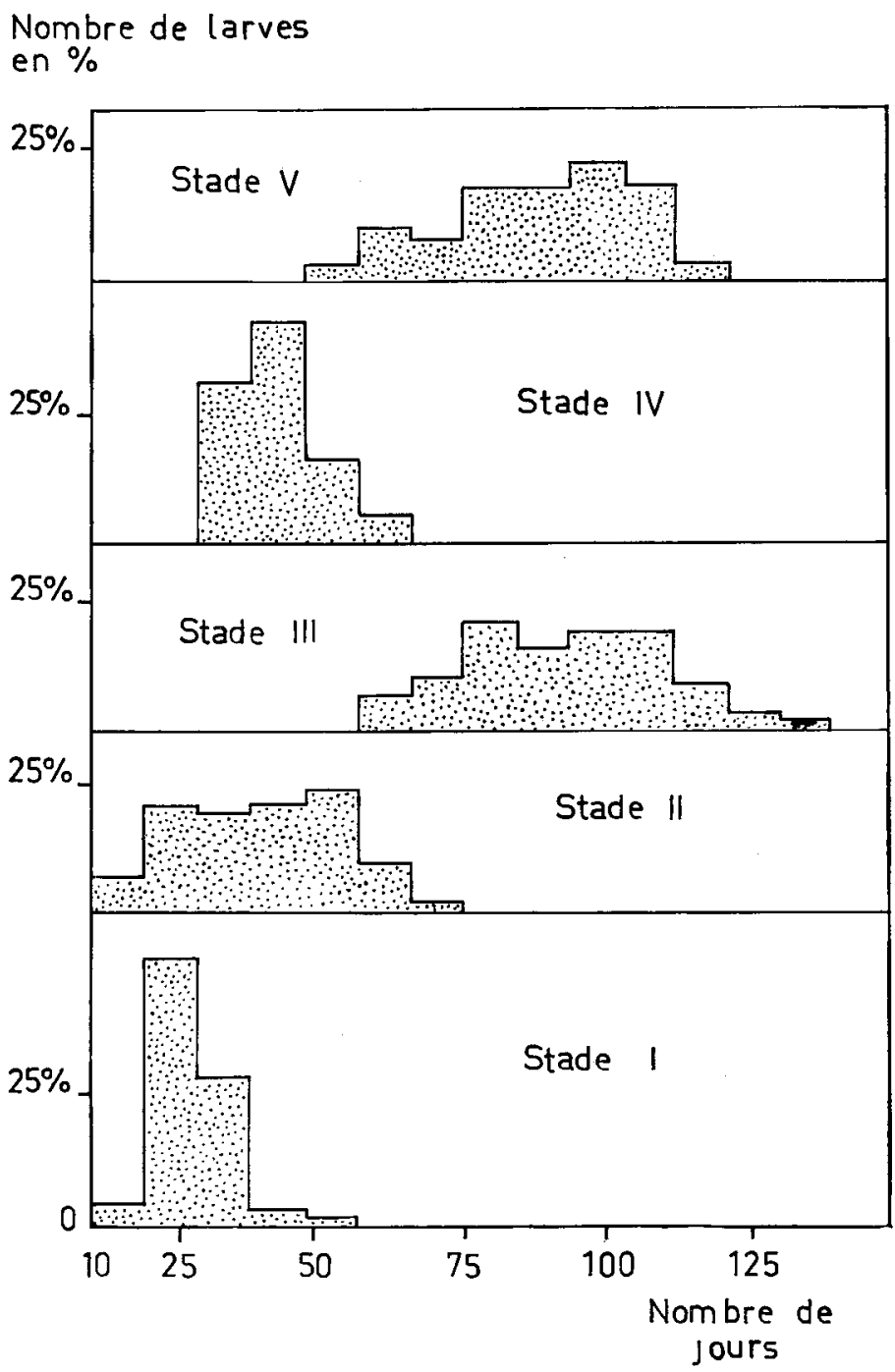

Fig. 1. - Variations de la durée du développement larvaire pour chaque stade des larves du groupe $A$. Intervalle des classe : 9 jours.

La durée de chaque stade varie considérablement selon les individus (Fig. 1). Elle est extrêmement importante pour le stade III en comparaison des autres stades; cet allongement est probablement dû à une sous-alimentation ou à un abaissement de la température des élevages $\left(9\right.$ à $\left.10^{\circ} \mathrm{C}\right)$ ou à l'effet combiné de ces deux facteurs. 


\section{2. — La vie nymphale.}

A la fin du $5^{\text {e }}$ stade, les larves abandonnent leur filet-piège et s'enferment dans un cocon où, après quelques jours, elles subissent la mue nymphale. La durée de vie nymphale, c'est-à-dire le temps compris entre la mue nymphale et la mue imaginale, varie avec la saison (fig. 2). Comme le montre la figure 2, cette variation semble dépendre de la température. C'est ce qu'avait noté KelneR-Pillault [1960] pour Enoicyla pusilla.

\section{3. - La vie imaginale.}

La nymphose achevée, la nymphe quitte son cocon, nage vers la surface, s'agrippe à un support émergé et subit, généralement en moins d'une minute, sa mue imaginale. Cette mue, contrairement aux mues larvaires et nymphale, semble toujours se produire la nuit. Alors que les adultes de certains Limnephilidae ont une vie imaginale relativement longue [Novak et Sehnal 1963, Bournaud travaux inédits], celle des imagos de $P$. conspersa est beaucoup plus brève. Elle est d'une dizaine de jours en moyenne pour les

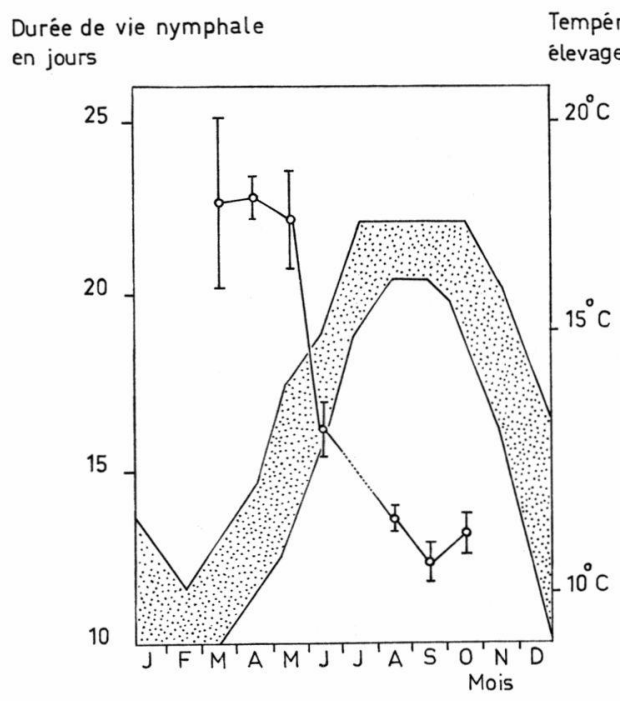

FIG. 2.

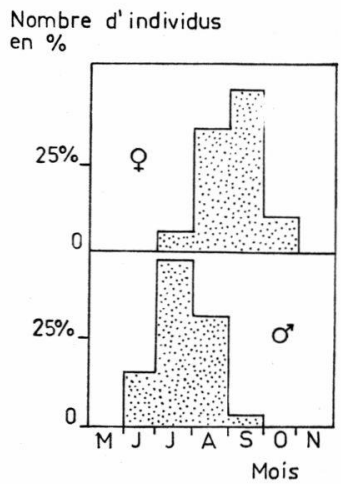

FIG. 3.

Fig. 2. - Variations de la durée de vie nymphale en fonction de la saison. Les valeurs moyennes sont calculées chaque mois, avec un intervalle de confiance pour $\mathrm{P}=0,05$. Ces résultats concernant à la fois des larves récoltées dans le Mornantet et des larves nées au laboratoire. La zone en grisé correspond aux variations de la température des élevages.

Fig. 3. - Nombre d'imagos $\sigma^{*}$ et + du groupe $A$ apparus chaque mois pendant l'été. 


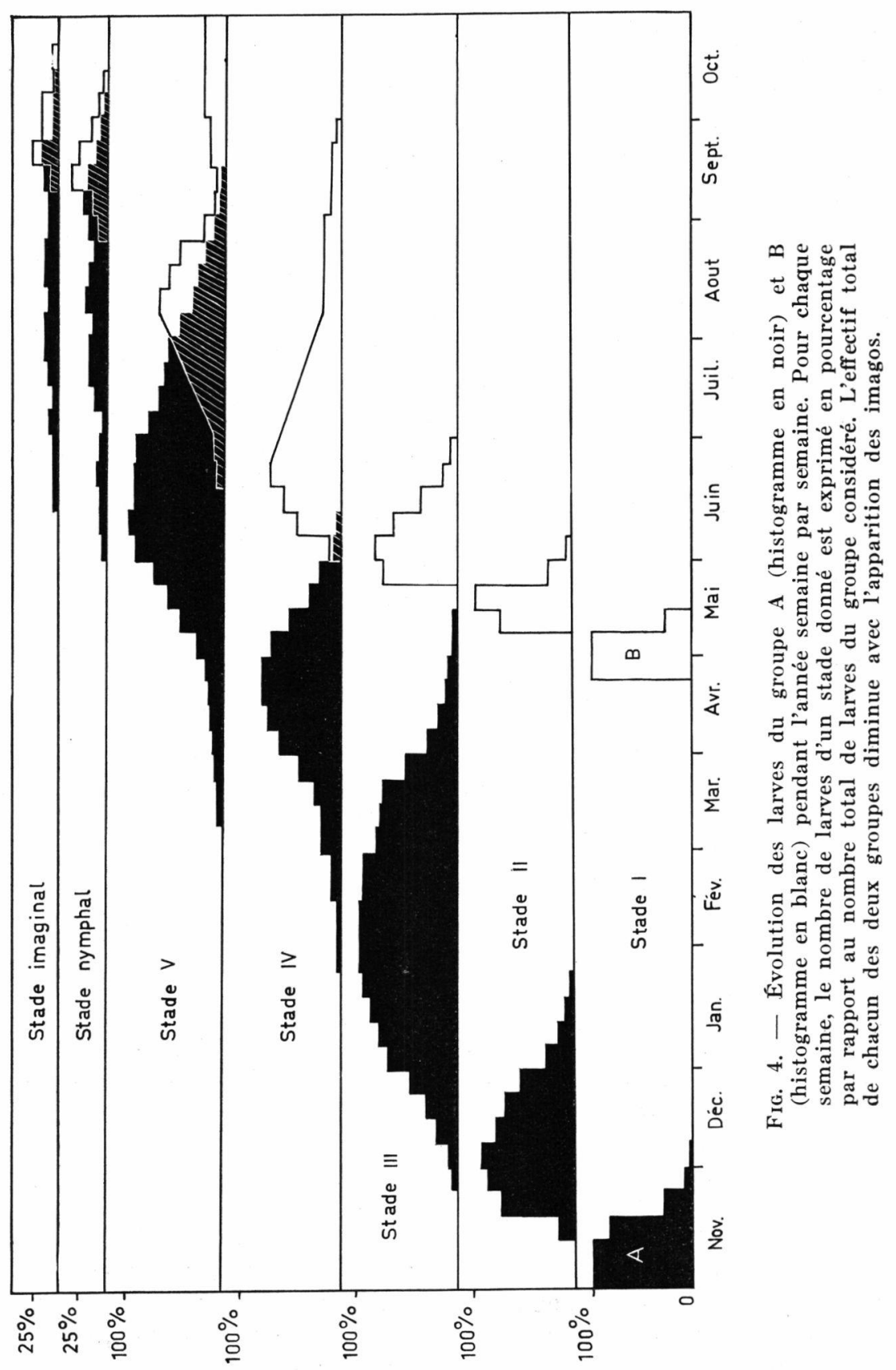


mâles et les femelles élevées à $18^{\circ} \mathrm{C}$ et sans nourriture. La maturation des ovaires est rapide puisque nous avons obtenu des pontes, dans deux cas, moins de quatre jours après la mue imaginale, et, dans un cas, moins de six jours après la mue imaginale. Sur 64 individus, nous avons obtenu 28 mâles et 36 femelles, soit $43 \%$ de mâles et $57 \%$ de femelles. Dans nos élevages, la majorité des mâles apparaissent avant les femelles (fig. 3). L'accouplement et la ponte n'ont pu être observés et doivent se produire la nuit.

\section{4. - La vie embryonnaire.}

La ponte a été bien décrite par Siltala [1907] et Nielsen [1942]. Nous avons observé des pontes de 110 à 420 œufs, ovoïdes, ayant $0,40 \times 0,35 \mathrm{~mm}$ au moment de la ponte. Le développement embryonnaire, aisément observable à travers l'enveloppe transparente de l'œuf, est immédiat et dure dans nos élevages 3436 jours à $12^{\circ} \mathrm{C}, 20-21$ jours à $16^{\circ} \mathrm{C}$ et $15-16$ jours à $18^{\circ} \mathrm{C}$. Nous n'avons obtenu aucun développement à partir de pontes vierges.

\section{3. - EVOLUTION DES LARVES DU GROUPE B.}

25 larves écloses le 24 avril 1966 furent mises en élevage; sur ce nombre, 23 seulement arrivèrent au dernier stade larvaire. Ces larves furent élevées dans les mêmes conditions que celles du groupe A, mais reçurent une nourriture plus abondante, composée de $10 \mathrm{~cm}^{3}$ environ du même milieu de culture que pour le groupe $\mathbf{A}$ pour le stade I et, respectivement, de 2, 3, 4, 6 Tubificides pour les stades II, III, IV et V.

Cette alimentation plus abondante et, peut-être, l'élévation de la température des élevages à cette période entraînent un abaissement important de la durée de vie larvaire $(140$ jours en moyenne), sensible à tous les stades (18 jours au stade 1,14 jours au stade II, 17 jours au stade III et 91 jours aux stade IV $+\mathrm{V}$ ). Nous n'avons observé aucun changement notable au niveau de la vie nymphale et de la vie imaginale, si ce n'est la production d'imagos plus petits.

\section{CONCLUSIONS.}

Sur la figure 4 se trouve rassemblée l'évolution des deux population $\mathrm{A}$ et $\mathrm{B}$. On peut constater que l'existence de deux générations est possible; la première correspondrait aux larves du groupe A nées à la fin de l'été ou au début de l'automne et qui passeraient l'hiver à l'état de larves de stade II ou III dans notre cas et, plus 
probablement, de stade $\mathrm{V}$ dans la nature. En effet, des larves provenant de la même ponte que celles du groupe $A$, mais élevées en groupe et ayant reçu une alimentation plus abondante, se trouvaient en majorité au stade $\mathrm{V}$ dès janvier. Ces larves atteindraient le stade imaginal à partir de mars. Les adultes pouvant pondre rapidement et le développement cmbryonnaire étant immédiat, on aboutirait à la formation d'une deuxième génération qui correspondrait au groupe $B$. Ce dernicr, à développement plus rapide, en raison peut-être d'une température plus élevée et d'une alimentation plus abondante à cette période (avril, mai, juin), atteindrait le stade imaginal en septembre - octobre et donnerait, avec les derniers individus du groupe $A$, une nouvelle génération.

Nos résultats sont encore trop fragmentaires et nos conditions d'élevage trop éloignées des conditions naturelles pour nous permettre de préciser quels sont les facteurs de milieu susceptibles d'influencer le cycle de $P$. conspersa. Il serait nécessaire d'analyser l'influence de la température, de l'alimentation et de la photopériode sur la durée de ce cycle vital.

\section{RESUME}

L'auteur a pu observer, au laboratoire, le cycle biologique de Plectrocnemict conspersa dans sa totalité. Cette espèce présente 5 stades larvaires. Les quatre phases principales de ce cycle sont analysées. L'influence de la température et de la nourriture sur ces 4 phases est discutée. Des températures élevées et une nourriture abondante entraînent un raccourcissement très important tu cycle biologique.

\section{SOME ASPECTS OF THE LIFE-CYCLE OF \\ PLECTROCNEMIA CONSPERSA (CuRTIS, 1834) \\ [Trichoptera, Polycentropodidae]}

The author was able to rear Pleclrocnemia conspersa under laboratory ronditions throughout its life-cycle. That species has 5 larval instars. The 4 main stages of its life-cycle are described. The influence of temperature and nutrition on these 4 stages is discussed. A warmer environment and plentyful feeding make the life-cycle considerably shorter.

\section{EINIGE ASPEKTE DES ENTWICKLUNGZYKLUS VON}

PLECTROCNEMIA CONSPERSA (CunTIS, 1834)

[Trichoptera, Polycentropodidae]

Der Autor konnte unter Laboratoriumsbedingungen den totalen Entwicklungzyklus von Plectrocnemia conspersa beobachten. Diese Art stellt 5 I.arvenstadien dar. Die 4 Hauptstadien des Entwicklungzyklus wurden analysiert. Der Einflu $B$ der Temperatur und der Nahrung auf diese 4 Stadien wurde besprochen. Hohe Temperaturen und reichliche Nahrung hatten zar Folge, den Entwicklungzyklus sehr bedeutend zu verkürzen. 


\section{TRAVAUX CITÉS}

Frost (W. E.). 1942. - River Liffey survey IV. The fauna of the submerged « mosses» in an acid and alkaline water. Proc. R. Irish. Acad., B 47 : 293-369.

Gower (A. M.). 1966. - The life cycle of Drusus annulatus Steph. (Trich. Limnophilidae) in watercress beds. Ent. Mon. Mag., 101 (1211$1213)$ : 133-141.

Hynes (H. B. N.). 1961. - The invertebrate fauna of a Welsh mountain stream. Arch. Hydrob., 57, $\mathrm{n}^{\circ} 3: 344-388$.

Kelner-Pillault (S.). 1961. - Biologie, écologie d'Enoicyla pusilla Burm. (Trichoptères Limnophilides). Année Biol., 36 (1/2) : 52-97.

Mackereth (J. C.). 1960. - Notes on the Trichoptera of a stony stream. Proc. R. ent. Soc. Lond., (A), 35 (1/3) : 17-23.

Nielsen (A.). 1942. - Über die Entwicklung und Biologie des Trichopteren mit besonderer Berücksichtigung der Quelltrichopteren Himmerlands. Arch. Hydrob., suppl., 17 : 255-631.

Novak (K.). 1959. — Entwicklung und Diapause der Köcherfliegen-larven Anabolia furcata BR. (Trichopt.). Čas. čsl. Spol. ent., 57 (3) : 207212.

Novak (K.) et Sehnal (F.). 1963. - The development cycle of some species of the genus Limnophilus (Trichoptera). Čas. čsl. Spol. ent., $60(1 / 2): 68-80$.

Siltala (A. J.). 1907. - Trichopterologische Untersuchungen. II. Über die post embryonale Entwicklung der Trichopteren-larven. Zool. Jber., suppl., 9 : 309-626.

(Laboratoire de Zoologie Générale, Faculté des Sciences, 16, quai Claude-Bernard, 69 - Lyon). 